\begin{abstract}
We developed a Ginzburg-Landau model of long-period stacking order (LPSO) lamellar structure observed in magnesium alloys. Contrary to other models, we do not treat LPSO as a homogeneous stoichiometric phase but as a chemically modulated heterogeneous structure of parallel plates of two phases with different compositions and degrees of ordering. The Turing instability that is, existence of a finite-wavelength linear-instability mode was identified as the origin of formation of LPSO structure. The process of LPSO formation can be understood as a process of finding local minima and saddle points on the free energy landscape of the system. The model explained transformation between the two most common LPSO structures, $18 \mathrm{R}$ and $14 \mathrm{H}$, and their general resistance to coarsening. The model can be used for the design of new LPSO materials.
\end{abstract}



in many different industries due to their excellent mechanical and thermal properties. This group of alloys is characterized by the structural order with long periods along the c-axis of the hexagonal closepacked planes of Mg matrix. This feature was called long-period stacking order (LPSO). All the alloys of the group have similarities in composition and processing that lead to formation of LPSO [1-8]. These are ternary Mg-TM-RE (TM-Transition Metal: zinc, copper, etc.; RE-Rear Earth: yttrium, gadolinium, etc.) alloys with a small overall concentration of the heavy elements. The distinct features of LPSO in Mg-2 at\% Y-1 at\% Zn-the most prominent member of the group-are the following [1-8]:

1. LPSO structures emerge either directly after the process of rapid solidification or/and after hot extrusion of the alloy. Both processes have large driving force of transformation.

2. The as-cast/extruded microstructures are composed of grains of two types and intermetallic particles along the grain boundaries. The Mg-Y grains have hcp structure and are featureless. The Mg-Y-Zn grains have fine-lamellar features with characteristic $18 \mathrm{R}$ structure.

3. LPSO is a strictly periodic lamellar structure of coexisting, alternating plates of hcp $\alpha-\mathrm{Mg}$ solid solution and fcc phase with intrinsic stacking faults of the close-packed planes.

4. There is strong synchronization between the periodicity of the lamellae and chemical modulations in the plates with the $\alpha-M g$ being almost completely void of the heavy elements and the fcc phase having practically fixed concentration of $\mathrm{Zn}$ and $\mathrm{Y}$ in the inner close-packed planes.

5. LPSO is practically a one-dimensional structure with the main variations in the c-direction of the $\alpha-\mathrm{Mg}$ crystalline lattice.

6. LPSO structures, characterized as 18R-type, are made of building blocks that have the same stacking orientations while those of $14 \mathrm{H}$-type are made of the building blocks that are in twin-orientation relationship.

7. 18R-type structure is highly resistant to change during heat treatment or overall variation in alloy composition. However, annealing at $500^{\circ} \mathrm{C}$ transforms the $18 \mathrm{R}$-type structure to the $14 \mathrm{H}$-type structure through the intermediate 24R-type structure. 


\section{GINZBURG-LANDAU MODEL OF LPSO STRUCTURE FORMATION}

1. Basic ideas

In this Section we propose a theoretical model of the LPSO structure of Mg-Zn-Y alloys. Main questions to be addressed by the model are: What is the phase content of the LPSO structure? Why is the structure strictly periodic and chemically synchronized? Why does this highly heterogeneous structure not coarsen quickly?

According to our model LPSO is not a phase but a two-phase structure, which consists of alternating plates of the disordered $\alpha-\mathrm{Mg}$ solid solution ( $\alpha$-phase) and ordered fcc phase with intrinsic stacking faults and composition $\mathrm{Mg}_{1-\mathrm{x} \beta}\left(\mathrm{Y}_{4} \mathrm{Zn}_{3}\right)_{\mathrm{x} \beta}(\beta$-phase). Alloying $\mathrm{Mg}$ (atomic radius $0.16 \mathrm{~nm}$ ) with $\mathrm{Y}$ (atomic radius $0.18 \mathrm{~nm}$ ) and $\mathrm{Zn}$ (atomic radius $0.14 \mathrm{~nm}$ ) in proportions close to $4 / 3$ practically removes the local strain since the average size of $(\mathrm{Y}+\mathrm{Zn})$ atoms is close to that of $\mathrm{Mg}$ [7]. This creates a virtual-solute species and a quasi-binary alloy with a very small amount of internal (built-in) stress. The remaining mismatch of atomic radii introduces shear strain between the atomic planes, which gives rise to the stacking faults in the crystalline lattice and two different orientations of the $\beta$-phase. In this regard, LPSO structure has strong similarities with the martensitic structure [6].

The LPSO structure forms in the precipitate particles in a process with large driving force of transformation. Formation of LPSO structure is not due to the mechanism of spinodal decomposition. It is rather a result of the so-called Turing instability when the structures emerge as a synchronous interplay of the two competing processes: in this case-ordering and diffusion [9-12]. Periodicity of the emerging structure in the precipitate particle is dictated by the most unstable mode of the fluctuations of order and composition. Initial period of the emerging structure grows because, overall, structural homogenization decreases the total free energy of the system. The 18R-type structure forms when the driving force for coarsening becomes not enough to overcome the barrier due to discreteness of the crystalline lattice. If the temperature of the system is raised, the free energies of the structures increase and the coarsening may resume, leading to the formation of a coarser structure with larger period $(14 \mathrm{H}-$ type). This process may be called LPSO coarsening.

\section{Phase-field theory of diffusional transformations}

In this Section, we recall the basic principles of the phase-field theory of diffusional transformations, which will be used below for the LPSO modeling $[13,14]$. The Gibbs free energy of a two-component system is expressed by a Ginzburg-Landau functional [15]: 


$$
G\{\eta, X\}=\int_{V} d^{3} x\left\{g(\eta, X)+\frac{1}{2} \kappa_{\eta}(\nabla \eta)^{2}+\frac{1}{2} \kappa_{X}(\nabla X)^{2}\right\}
$$

where $g$ is the Gibbs free energy density of a homogeneous system (we consider its molar density to be constant), $\eta(\mathbf{x}, \mathrm{t})$ and $\mathrm{X}(\mathbf{x}, \mathrm{t})$ are, respectively, continuously distributed order parameter (OP) and concentration fields, $\kappa_{\eta}$ and $\kappa_{x}$ are the order-parameter and composition gradient-energy coefficients and $\mathrm{V}$ is the volume. Dynamics of the system is described by the time-dependent Ginzburg-Landau equation (TDGLE) for the order-parameter field evolution:

$$
\frac{d \eta}{d t}=-\gamma \frac{\delta G}{\delta \eta}
$$

and Cahn-Hilliard equation (CHE) for the evolution of the concentration field [14, 15]:

$$
\frac{d X}{d t}=\nabla M \nabla \frac{\delta G}{\delta X}
$$

where $\gamma$ is the relaxation coefficient and $M$ is the species mobility.

Equilibrium states of the system described by Eqs.(1, 2) satisfy equations: $\delta G / \delta \eta=\delta G / \delta X=0$. As known $[13,14]$, the set of homogeneous solutions of these equations contains phases of the system and the potential barriers, represented by the saddle points. In this work we are looking at a system consisting of two phases, e.g., $\alpha$-phase $\left(\eta_{\alpha}, X_{\alpha}\right)$ and $\beta$-phase $\left(\eta_{\beta}, X_{\beta}\right)$ where the latter may have two orientation variants: $\beta_{+}$and $\beta_{-}$. The heterogeneous solutions of the equilibrium equations can be classified based on their dimensionalities. The set of one-dimensional (1D) solutions contains equilibrium two-phase interfaces, critical plates, and periodic solutions, sometimes called periodons. (2D and 3D solutions will not be of interest in this publication.) An interface is a transition zone of finite thickness. A critical plate is a localized excitation of order of finite amount. A periodon consist of interacting plates of finite thickness. All 1D equilibrium structures are characterized by the free-energy excess per unit area measured in the direction perpendicular to the direction of variation of the structures (the c-direction in our case) [14]:

$$
\sigma_{1 D}=\int_{-\infty}^{+\infty}\left\{\kappa_{\eta}\left(\frac{d \eta}{d x}\right)^{2}+\kappa_{X}\left(\frac{d X}{d x}\right)^{2}\right\} d x
$$

For the two-phase equilibrium interface, this amounts to the interfacial energy $\sigma$. The free-energy excess makes the periodic structures unstable and gives rise to the coarsening mechanism, which is significantly different from the traditional Lifshitz-Slyozov one. The driving force of the latter is the 
curvature of the interphase interfaces; it vanishes when the interfaces are flat. The driving force of the former is the free-energy excess itself; it exists even when all interfaces are flat. The difference in the driving forces leads to the difference in structural evolutions of the systems.

The homogeneous equilibrium states of the system may be stable, metastable or unstable. Transformation out of a metastable state proceeds via a process of nucleation. An unstable state decomposes, so to speak, by itself that is, due to small fluctuations, which are always present in the system. To examine initial stages of the decomposition of a homogeneous unstable equilibrium state $\left(g_{\eta \eta}<0\right)$, we superimpose on it a disturbance in the form of a linear mode $\{\Delta \eta, \Delta X\}=\{H, \Theta\} \exp (\beta t+i \mathbf{k} \cdot \mathbf{x})$ where $\beta$ is an amplification rate and $\mathbf{k}$ is a 3D wave-vector, substitute it into Eqs.(2), linearize them and find that, for the mode to be a solution, the following characteristic equation must be satisfied:

$$
\left(\frac{\beta}{\gamma}+\kappa_{\eta} k^{2}+g_{\eta \eta}\right)\left\{\frac{\beta}{M}+\left(\kappa_{X} k^{2}+g_{X X}\right) k^{2}\right\}=g_{\eta X}^{2} k^{2}
$$

where $k=|\mathbf{k}|$. The characteristic equation (4) has two branches. If $g_{\eta x}=0$, these are the transformation branch $\beta_{\gamma}=-\gamma\left(g_{\eta \eta}+\kappa_{\eta} k^{2}\right)$ and diffusive branch $\beta_{M}=-M\left(g_{x x}+\kappa_{\eta} k^{2}\right) k^{2}$ that is, the processes proceed independently. Spinodal decomposition is an important limiting case of this regime when $\mathrm{g}_{\mathrm{xx}}<0$ (miscibility gap) and $\kappa_{\chi} \neq 0$ (uphill diffusion). If $g_{\eta \chi} \neq 0$, the transformation and diffusion processes interact and the branches morph into $\beta_{+}(k)$ and $\beta_{-}(k)$ with $\beta_{+}(k) \geq \beta_{-}(k)$. The more important branch $\beta_{+}(k)$ has the following characteristic modes: the uniform $\left(k_{0}=0, \beta_{0} \gtrless 0\right)$, the cutoff $\left(k_{c} \neq 0, \beta_{c}=0\right)$, and the most unstable $\left(\mathrm{k}_{\mathrm{T}}, \beta_{\mathrm{T}}=\mathrm{max}\right)$. The latter dominates initial structure formation during decomposition of an unstable homogeneous system. The case when the most unstable mode is not uniform that is, $\mathrm{k}_{\mathrm{T}}>0$ is called Turing instability $[9,10]$.

Let us look at the decomposition of unstable states $\left(g_{\eta \eta}<0\right)$ of a binary system without the miscibility gap $\left(g_{x x}>0\right)$. In such system the uphill diffusion is not important and we can disregard the concentration gradient-energy coefficient $\left(\kappa_{\mathrm{x}}=0\right)$. Then the uniform and cutoff modes are characterized by:

$$
\begin{aligned}
& \beta_{0}=-\gamma g_{\eta \eta} \\
& k_{c}^{2}=-\frac{g_{\eta \eta}}{\kappa_{\eta}}(A+1) \\
& A \equiv-\frac{g_{\eta X}^{2}}{g_{\eta \eta} g_{X X}}
\end{aligned}
$$


where the module $A$ is a measure of the strength of interactions between the ordering and diffusion. Analysis of the characteristic equation, Eq.(4), shows that the Turing mode exists if:

$$
\begin{aligned}
& R A>1 ; \\
& R \equiv \frac{M g_{X X}}{\gamma \kappa_{\eta}}
\end{aligned}
$$

where the number $R$ is a ratio of the diffusion coefficient $D=M g_{x x}$ and interfacial mobility $m=\gamma \kappa_{\eta}$. Then we obtain an expression for the Turing mode:

$$
\frac{\kappa_{\eta}}{\left(-g_{\eta \eta}\right)} k_{T}^{2}= \begin{cases}\frac{A^{2}-1}{4 A}, & \text { if } R=1 \\ \frac{1-R-2 R A \pm(1+R) \sqrt{A(R A+R-1)}}{(1-R)^{2}}, & \text { if } R \neq 1\end{cases}
$$

Criterion Eq.(6) shows that existence of the Turing instability for Eqs.(2) requires a combination of fast diffusion and/or strong coupling between the ordering and species distribution. Notice that this case, Eqs. $(6,7)$, is similar to the spinodal decomposition in that the Turing mode is not the uniform one, but the role of the concentration gradient-energy coefficient is played by $\kappa_{\eta}\left(g_{x x} / g_{\eta x}\right)^{2}$. In Figure 1 is depicted the case when $R=1$ and $A=5$.

3. The order parameter and the free energy

In the above described model, the transformation between the disordered $\alpha$-phase and ordered $\beta$ phase is a combination of displacive deformation and chemical ordering where the shear strain of the close-packed planes plays the role of the order parameter. To be exact, the order parameter of the transformation $\eta$ is the ratio of the actual shear strain $\varepsilon$ and the stress-free transformation shear strain $\varepsilon_{\beta}$ between the hcp $\alpha-\mathrm{Mg}$ and fcc $\beta$-phase with the intrinsic stacking faults. As positive and negative values of the shear stain are equally probable, the free energy of the system should be expressed by an OP-symmetric potential. For a 2-4-6 Landau potential the OP values of the equilibrium phases vary with the variations of the control parameters: temperature, pressure, concentration, etc. This is not the case for the LPSO structure (see property 7 in Sec.I). To describe transformations where the OP's of the equilibrium phases do not change much with the change of the control parameters, one of the authors introduced a group of potentials, which he called 'tangential' $[13,17]$. Using these ideas, the Gibbs free energy density of a homogeneous system may be expressed as follows: 


$$
g(\eta, X ; S)=g_{\alpha}(X)+\frac{1}{2} W \omega^{2}(\eta)+\Delta g(X) v(\eta)-S \eta
$$

Here, the first term $g_{\alpha}(X)$ expresses the Gibbs free energy density of a homogeneous $\alpha$-phase, the second term expresses the free energy barrier between the $\alpha$-and $\beta$-phases where $W$ is the scale of the barrier height, the third term biases one phase against the other with $\Delta g \equiv g_{\beta}(X)-g_{\alpha}(X)$ being the free energy difference between the $\beta$ - and $\alpha$ - phases, and the fourth term is the work done on the system by the external force $S$ conjugate to the OP. In the present model $S$ is assumed to be the shear stress normal to the c-direction. In this study, the following expressions were used (see Figure 2):

$$
\begin{gathered}
g_{\alpha}(X)=B_{0}+B_{1} X+\frac{1}{2} U X^{2} \\
\Delta g(X)=-U[X]\left(X-X_{\alpha \beta}\right) \\
\omega(\eta)=\eta\left(1-\eta^{2}\right) ; \\
v(\eta)=\frac{1}{\Omega} \int_{\alpha}^{\eta} \omega\left(\eta^{\prime}\right) d \eta^{\prime}=\eta^{2}\left(2-\eta^{2}\right) ; \\
\Omega=\int_{\alpha}^{\beta} \omega(\eta) d \eta=\frac{1}{4} ;
\end{gathered}
$$

In Eqs. $(8 a, b) U=g_{x x}$ is the compositional 'stiffness' of the alloy, assumed to be equal in the $\alpha$ - and $\beta$ phases; $[X]=X_{\beta}-X_{\alpha}$ is the equilibrium concentration range and $X_{\alpha \beta}=\left(X_{\beta}+X_{\alpha}\right) / 2$ is the middle of the range. In a system free of the external stresses $(S=0)$, the order parameters of the stable phases, $\eta_{\alpha}=0, \eta_{\beta+}=+1$, and $\eta_{\beta-}=-1$, do not vary with the variation of the concentration of the system. The stable phases are separated by the barrier states whose order parameters vary with the variation of the concentration according to the following relation:

$$
3 \eta_{B}^{2}=1-\frac{U[X]}{\Omega W}\left(X-X_{\alpha \beta}\right)
$$

Intersections of the barrier-state and phase lines give rise to the respective spinodal points $S_{\alpha}=(0$, $\left.\mathrm{X}_{\alpha \beta}+\Omega \mathrm{W} / \mathrm{U}[\mathrm{X}]\right)$ and $\mathrm{S}_{\beta \pm}=\left( \pm 1, \mathrm{X}_{\alpha \beta}-2 \Omega \mathrm{W} / \mathrm{U}[\mathrm{X}]\right)$. The criterion of existence of the Turing mode, Eq.(6), applied to the potential, Eq.(8), takes the form: 
Eq.(10) shows that there is a limited range of concentrations $X$ of the alloy where the Turing mode exists. The special points and lines of the stress-free system described by Eqs.(8) are shown in Figure 3. Application of the external stress changes the OP values of the equilibrium phases and the barrier states. For small values of the stress the changes are linearly proportional as given by:

$$
\begin{gathered}
\eta_{\alpha}(S)=\frac{\Omega S}{\Omega W+\Delta g(X)} \\
\eta_{\beta \pm}(S)= \pm 1+\frac{\Omega S}{W-2 \Delta g} \\
\eta_{B \pm}(S)=\eta_{B \pm}(0)-\frac{3 \Omega^{2} W S}{(\Omega W+\Delta g)(W-2 \Delta g)}
\end{gathered}
$$

Notice that the equilibrium phases undergo shifts in the direction of the applied stress while the barrier states-in the opposite direction.

\section{Parameters of the model}

The model described by Eqs. $(1,2,8)$ has ten material parameters out of which there are five thermodynamic macroscopic parameters: $B_{0}, B_{1}, U, X_{\alpha}$ and $X_{\beta}$, three mesoscopic GL parameters: $W, \kappa_{\eta}$ and $\kappa_{\mathrm{x}}$, and two kinetic parameters: $\gamma$ and $\mathrm{M}$, and two control parameters: the overall concentration $\bar{X}$ and external stress S. For a theoretical model to be relevant to experimental observations, the model's material parameters must be calibrated with respect to the physical parameters of the system of interest. The macroscopic parameters were obtained from the CALPHAD $[18,19]$ and DICTRA databases (see Figure 4). The values of the essential for the model parameters are listed in Table 1.

Table 1

\begin{tabular}{|l|l|l|l|l|l|l|l|l|l|l|}
\hline$\mu$ & $\varepsilon_{\mathrm{m}}$ & $\mathrm{U}$ & $\mathrm{X}_{\alpha}$ & $\mathrm{X}_{\beta}$ & $\mathrm{D}$ & $l_{\text {LPSO }}$ & $\mathrm{W}$ & $\mathrm{Q}$ & $\mathrm{K}$ & $\mathrm{R}$ \\
\hline $\mathrm{J} / \mathrm{m}^{3}$ & $\mathrm{frac}$ & $\mathrm{J} / \mathrm{m}^{3}$ & $\mathrm{frac}$ & $\mathrm{frac}$ & $\mathrm{m}^{2} / \mathrm{s}$ & $\mathrm{nm}$ & $\mathrm{J} / \mathrm{m}^{3}$ & -- & -- & -- \\
\hline $17 \times 10^{9}$ & $1 / 2 \pi$ & $7.206 \times 10^{9}$ & 0.05 & 0.25 & $1 \times 10^{-14}$ & 1.5 & $1.6 \times 10^{8}$ & $0.2 \div 5$ & $0.125 \div 5$ & $\sim 1$ \\
\hline
\end{tabular}


To obtain the mesoscopic GL parameters one needs to calculate certain physical properties and compare them with the same properties calculated from the first principles ( $a b$ initio, DFT, MD, etc.) or measured experimentally. To find the GL barrier-height parameter W and gradient-energy coefficients one usually calculates theoretically the energy and thickness of the $\alpha / \beta$-interphase interface and compares them with the respective experimental values. Because the LPSO interfacial energy is not readily available, we suggest another way to estimate the GL parameter W. First, we notice that when the order parameter value is small our model describes elastic shear deformation of the $\alpha-M g$ phase $\left(X=X_{\alpha}\right)$ :

$$
g\left(\varepsilon, X_{\alpha} ; 0\right) \approx g_{\alpha}\left(X_{\alpha}\right)+\left(\frac{1}{2}+Q\right) W\left(\frac{\varepsilon}{\varepsilon_{\beta}}\right)^{2}
$$

where we introduced the ratio of the energy density scales as

$$
Q \equiv \frac{U[X]^{2}}{W}
$$

Second, we may assume that the order parameter of the barrier state at $\mathrm{X}=\mathrm{X}_{\alpha}$ represents the critical shear strain $\varepsilon_{m}$ of the theoretical shear strength of the $\alpha-M g$ phase [20]. Then, using Eq.(9) we obtain:

$$
\left(\frac{\varepsilon_{m}}{\varepsilon_{\beta}}\right)^{2}=\eta_{B}^{2}\left(X_{\alpha}\right)=\frac{1}{3}\left(1+\frac{Q}{2 \Omega}\right)
$$

Eliminating $\varepsilon_{\beta}$ from Eqs. $(12,13)$ we obtain an expression for the free energy of the $\alpha-\mathrm{Mg}$ phase at small deformations:

$$
g\left(\varepsilon, X_{\alpha} ; 0\right) \approx g_{\alpha}\left(X_{\alpha}\right)+\frac{(1+2 Q)^{2} W}{6 \varepsilon_{m}^{2}} \varepsilon^{2} .
$$

The coefficient in front of the square of the shear deformation in this formula is the shear modulus $\mu$ [21]. Hence,

$$
\mu=\frac{(1+2 Q)^{2} W}{6 \varepsilon_{m}^{2}}
$$

Taking into account Eq.(12a) we obtain an equation for Q:

$$
Q+\frac{1}{4 Q}+1=\frac{3 \mu \varepsilon_{m}^{2}}{2 U[X]^{2}}
$$

Eq.(15) tells us that for the above described model to be valid the following constraint must be fulfilled: 


$$
\frac{\mu \varepsilon_{m}^{2}}{U[X]^{2}}>\frac{4}{3}
$$

If $U[X]^{2} \approx \mu \varepsilon_{m}^{2}$ then $\mathrm{W} \approx 2 \mathrm{U}[\mathrm{X}]^{2}$; if $U[X]^{2} \ll \mu \varepsilon_{m}^{2}$ then the solution of Eq.(15) has two branches: small$Q$ and large- $Q$ where

$$
W \approx \begin{cases}6 \mu \varepsilon_{m}^{2}, & \text { if } Q<1 / 2 \\ \frac{2\left(U[X]^{2}\right)^{2}}{3 \mu \varepsilon_{m}^{2}}, & \text { if } Q>1 / 2\end{cases}
$$

Based on the analysis of the stable phases in the ternary Mg-Y-Zn system [22], we estimate $X_{\alpha}=0.05$ and $\mathrm{X}_{\beta}=0.2$. Then (see Table 1$) \mu \varepsilon_{m}^{2} / U[X]^{2} \approx 2.4$. Furthermore, analysis of Eq.(10) shows that the criterion of existence of the Turing mode favors the large-Q branch of Eq.(17). Hence, we arrive at the following estimate of the relevant energy scales of the system: $W \approx U[X]^{2} \approx \mu \varepsilon_{m}^{2} / 2 \approx k_{B} T_{\text {room }}$ that is, the LPSO forming systems have relatively low free energy barriers.

In order to obtain both the order-parameter and composition gradient-energy coefficients, $\kappa_{\eta}$ and $\kappa_{x}$ one may introduce the length scales of the order parameter and concentration distributions:

$$
\begin{gathered}
l_{\eta}^{2}=\frac{\kappa_{\eta}}{W}, \quad l_{X}^{2}=\frac{\kappa_{X}}{U}, \\
K \equiv \frac{l_{X}^{2}}{l_{\eta}^{2}}=\frac{\kappa_{X} W}{\kappa_{\eta} U}
\end{gathered}
$$

and compare them to the experimentally resolved distributions of the ordering and concentration. Unfortunately the available experimental data [1-8] allow us to find only the combined LPSO interfacial thickness of $l_{\text {LPSO }}=1.5 \mathrm{~nm}$. We assume that it corresponds to the order-parameter length scale of $l_{\eta}$; this allows us to find the order-parameter gradient-energy coefficient $\kappa_{\eta}$. Although LPSO formation is not a spinodal decomposition process and we do not expect the concentration-gradient coefficient $\kappa_{\mathrm{x}}$ to play significant role, for generality, we retain the latter in the model.

The dynamic equation, Eq.(2a), and the free-energy density, Eq.(8), motivate the following time scale:

$$
\tau_{\eta}=\frac{1}{\gamma W}
$$


The relaxation coefficient $\gamma$ can be obtained from the kinetic data on the LPSO formation, which were not available to the authors. The lack of required data prompted us to conduct simulations with various values of the dimensionless material parameters Q, Eq.(12a), K, Eq.(18a), and R, Eq.(6a), (see Table 1). Notice that ten dimensional material parameters are reduced to only three dimensionless ones. Also the simulation results depend on the dimensionless overall concentration:

$$
\bar{C}=\frac{\bar{X}-X_{\alpha}}{[X]}
$$

and dimensionless shear stress:

$$
\Sigma=\frac{S}{W}
$$

\section{SIMULATION RESULTS}

In this publication we present results of the simulations of LPSO formation in the 1D system because LPSO is a practically 1D structure where most significant variations take place in the direction parallel to the c-direction of the crystalline planes. Evolution of the system is described by the dynamic equations in the volume $V=1 \times 1 \times 250$ where the length and time were measured respectively in the units of $l_{\eta}$, Eq.(18), and $\tau_{\eta}$, Eq.(19). Eqs.(1, 2, 8) were solved for the dimensionless fields of the order-parameter $\eta(\mathrm{x}, \mathrm{t})$ and concentration $C(x, t)=\left(X(x, t)-X_{\alpha}\right) /[X]$ using explicit finite-differences scheme based on a one-dimensional five-point space and two-point time grid with $\Delta \mathrm{x}=0.5$ and $\Delta \mathrm{t}=0.001$. As a verification of the method the mass balance was checked at each time step; it was better than $1 \%$.

Initially, a compositionally homogeneous system of the overall concentration $C=\bar{C}$ occupied one of the two barrier states (see Eq.(9)) and a small amount of noise was added to it. Such choice of the initial conditions can be justified by the large driving force of the experimental processing leading to LPSO formation (see property 1 in Sec.I). In the beginning, Stage 1 (see Figure 5a), we observed rapid growth of the Turing mode, which ended on Stage 2, (see Figure 5b). After the initial stage, a periodic structure has formed in the system. The wave-vector of the structure started decreasing immediately after its formation, which means that the structure coarsened (Stage 3, see Figure 5c). When the orderparameter increment $\Delta \eta$ dropped below the level where $(\delta G \eta(x, t) / \delta \eta) \Delta t \leq 3 \times 10^{-8}$ the coarsening stopped and the structure remained without any change. The number $\delta_{m}=3 \times 10^{-8}$ is called the floating point precision [23]; it represents the low limit for the numerical calculations. 
In Figure 6 is depicted the structure formed at $\mathrm{Q}=1, \mathrm{~K}=1, \mathrm{R}=1, \bar{C}=0.5$, and $\Sigma=0$. One can recognize the distinct features of the 18R-type structure: periodicity and gradual change of the ordering and composition distributions. The structure depicted in Figure 7 was formed by initially periodically distributing in equal proportions the OP of the system with the same parameter values as in Fig. 6 on both barriers of the free energy potential. One can recognize in Fig. 7 the distinct feature of the $14 \mathrm{H}-$ type structure: the opposite ordering of the $\beta$-plates. Other important characteristics of the $14 \mathrm{H}$-type structure that differ from the 18R-type are the greater spacing of $\alpha$-plates and slightly higher average concentrations of the $\beta$-plates. All of the $14 \mathrm{H}$ interfaces were of the type $\alpha / \beta_{+}$or $\alpha / \beta_{-}$; the high-energy $\beta_{+} / \beta_{-}$interfaces did not appear in the simulated systems. In Fig. 3 the same structure as in Figs. 6, 7 are depicted on the phase map that is, in the $(\eta, C)$-phase plane. The trajectories in this plane represent the tie lines of the phase diagram. By varying the initial periodicity, the numerical experiments produced structures depicted in Figure 8, which may be identified as 18R-twin structure.

The total free energy of a structure (per unit area) decreased with time until the moment when the order-parameter increment dropped down to the level of the floating-point precision. During that period of time the total free energy of the $14 \mathrm{H}$-type structure decreased to the level lower than that of the 18R-type structure (see Figure 9). Hence, the free energy difference of the structures constitutes the driving force of the $18 \mathrm{R} \rightarrow 14 \mathrm{H}$ transformation. The total free energies of the structures should be reckoned with the interfacial energy $\sigma$ (see Fig. 9).

Main characteristics of the emerging structures are the free-energy density excess $\delta g$, length $L$ and $\beta$ phase fraction $\mathrm{F}$ of the structural periods. The free-energy density excess is The length of the period $L$ is calculated as a quotient of the system size in the c-direction and the number of the $\beta$-plates, combining the number of $\beta_{+}$and $\beta_{-}$plates for the $14 \mathrm{H}$ structure. The $\beta$-phase fraction $\mathrm{F}$ is the ratio of the lengths of the $\beta$-plate and the whole period (half-period for the $14 \mathrm{H}$ structure). In Figures 10-12 these characteristics are presented as functions of the control and material parameters of the system $\mathrm{Q}, \mathrm{K}$, and $\bar{C}$, for the values which satisfy the condition of existence of the Turing mode, Eq.(10): $R Q[1-$ $Q(1-2 \bar{C})]>(3 \Omega)^{2}$. Figure 10 shows that the driving force of the $18 \mathrm{R} \rightarrow 14 \mathrm{H}$ transformation increases with the increase of the energy parameter $Q$. This confirms our conclusion that the large- $Q$ branch of Eq.(17) is realized in the LPSO forming systems.

The overall concentration $C=\bar{C}$ also has strong influence on the LPSO structural characteristics and the $18 \mathrm{R} \rightarrow 14 \mathrm{H}$ transformation. Figure 11 shows that the driving force of the $18 \mathrm{R} \rightarrow 14 \mathrm{H}$ transformation is at maximum in the middle of the $\alpha-\beta$ concentration range and that the optimal for the transformation 
conditions produce the smallest length of the period. Not surprisingly Fig. 11 shows that the $\beta$-phase fraction of the period is linearly proportional to the overall concentration $C=\bar{C}$. Fig. 11 and Table 1 allow us to estimate the maximum value of the driving force of the $18 \mathrm{R} \rightarrow 14 \mathrm{H}$ transformation as 0.5 meV/atom.

Figure 12 shows that variation of the length of the structural period in the whole range of the variation of the overall composition decreases with the decrease of the gradient-energy number K. Given the fact that LPSO structures are highly resistant to change due to variation of the overall composition, we conclude that in real systems the number $\mathrm{K}$ is small.

Our model allows us to study reaction of LPSO on the shear stress applied parallel to the lamellae of the structure that is, perpendicular to the c-direction of the crystalline lattice. In Figure 13 are depicted dependencies of the structural parameters of LPSO on the shear stress. One can see that the applied stress 'quickly' destroys the LPSO. The 18R-type LPSO structure was completely eliminated at $\Sigma_{18 R} \approx \pm 0.05$ and the homogeneous phases were restored: $\alpha$-phase at $\Sigma_{18 \mathrm{R}}=0.04$ and $\beta$-phase at $\Sigma_{18 \mathrm{R}}=-0.05$. The $14 \mathrm{H}$ type LPSO structure happened to be even more sensitive to the applied shear stress -it was completely eliminated at $\Sigma_{14 \mathrm{H}} \approx \pm 0.002$. Such sensitivity of LPSO to the shear stress is surprising given that the shifts of the equilibrium points due to these magnitudes of stress are relatively small, see Eqs.(11). The definition of the order parameter, relation in Eq.(13), and Table 1 allow us to estimate the critical stress that destroys the $18 \mathrm{R}$ LPSO structure as $\Sigma_{18 \mathrm{R}} \varepsilon_{\mathrm{m}} \mathrm{W}(3 / 1+2 \mathrm{Q})^{1 / 2} \approx 10^{4} \mathrm{~Pa}$.

\section{DISCUSSION AND CONCLUSIONS}

In this work, we constructed a phase-field model of structure formation in quasi-binary alloys. The model describes evolution of the system governed by Eqs. $(2,3)$ for the Gibbs free energy functional, Eqs. $(1,8)$. Formation of the structure and its subsequent coarsening is dictated by minimization of the total free energy of the system, which incorporates the bulk and interfacial contributions. The model was able to reproduce different types of LPSO structure observed in real magnesium alloys, which are characterized by seven basic properties described in Sec. I. The LPSO was identified not as a homogeneous phase but as a heterogeneous, two-phase structure of the ternary $\mathrm{Mg}-\mathrm{X}^{\mathrm{S}}-\mathrm{X}^{\mathrm{L}}$ alloy with coexisting hcp $\alpha-\mathrm{Mg}$ and fcc phase with intrinsic stacking faults. Combination of the theoretical analysis and numerical simulations helped us identify the Turing instability that is, existence of a finite-wavelength linear-instability mode, as the origin of the LPSO formation. Followed by rapid solidification and/or extrusion, the alloy produces grains with enough concentration of heavy elements for the Turing instability, which creates a strictly periodic and highly synchronized structure. In other words, LPSO is an 
example of Turing-like patterns, which are plentiful in the natural world [24]. It is important to note that the ordering process alone does not produce the Turing instability because the most unstable mode of that process is uniform.

The process of LPSO structure formation takes place in the alloy precipitate particles and can be divided into three stages. Stage 1 starts in a highly disordered unstable state and passes to Stage 2-the linear regime of structure formation. On Stage 3, the linear regime of the structure formation is replaced by the nonlinear adjustment of the structure (LPSO coarsening). On this stage of the process, we observed the structures similar to LPSO 18R-type-all plates of fcc phase have same orientation and 14H-typefcc plates have alternating orientation; occasionally 18R-twin LPSO structures were produced in the system. The 18R-type and 14H-type structures also differ in the density of the interfaces-number of interfaces per unit length in the c-direction. This yields the free energy difference between the $18 \mathrm{R}$ and $14 \mathrm{H}$ structures, which is the driving force of the $18 \mathrm{R} \rightarrow 14 \mathrm{H}$ transformation. On Stage 3 of the process, the structure coarsening slows down and terminates completely. Numerically, the coarsening was blocked by the floating-point precision $\delta_{\mathrm{f}}=3 \times 10^{-8}$. In real alloys the coarsening of LPSO structures is blocked by the interatomic barriers of the crystalline lattice. Thus, the floating-point precision $\delta_{\mathrm{f}}$ can be called the "numerical Peierls barrier".

In the presented model we have two essential energy density scales, the barrier height $W$ and the chemical scale $U[X]^{2}$, which should be compared not only between themselves but also with the energy density of the theoretical shear strength, $\mu \varepsilon_{m}^{2}$. The theoretical analysis revealed the following estimate of the relevant energy scales: $W \approx U[X]^{2} \approx \mu \varepsilon_{m}^{2} / 2 \approx k_{B} T_{\text {room. }}$. Dimensional analysis helped us identify the energy parameter $\mathrm{Q}$, length scale parameter $\mathrm{K}$, and kinetic number $\mathrm{R}$ as the dimensionless material parameters, and the overall composition $\bar{C}$ and the applied stress $\Sigma$ as the control parameters of the process in the system. Analysis of the main structural characteristics-free energies of the $18 \mathrm{R}$ and $14 \mathrm{H}$ structures, the size and composition of the periodic plates-showed that the driving force of the $18 \mathrm{R} \rightarrow 14 \mathrm{H}$ transformation increases with the increase of the energy parameter $\mathrm{Q}$ and is at maximum in the middle of the $\alpha-\beta$ concentration range. The maximum value of the driving force of the $18 \mathrm{R} \rightarrow 14 \mathrm{H}$ transformation was estimated as $0.5 \mathrm{meV} /$ atom. The optimal for the transformation conditions were found to correspond to the smallest size of the period. Shear stress applied parallel to the lamellae has destructive effect on the LPSO structure. We found that small amount of shear $\left(\Sigma_{18 \mathrm{R}} \approx \pm 0.05\right)$ eliminates the 18R-type LPSO; even smaller amount of shear $\left(\Sigma_{14 \mathrm{H}} \approx \pm 0.002\right)$ was required to destroy the delicate 
symmetry of the $14 \mathrm{H}$-type LPSO. The critical stress that destroys the $18 \mathrm{R}$ LPSO structure was estimated as $10^{4} \mathrm{~Pa}$. These results can be used for the design of new LPSO materials.

The process of LPSO formation can be understood as a process of finding a local minimum or a saddle point on the free energy landscape of the system [13-15]. The landscape has different domains: homogeneous, one-well inhomogeneous (18R-type), and two-well inhomogeneous (14H-type). The process starts on the barrier of the homogeneous part of the landscape. At first, it is driven by the Turing instability and it lands on the landscape close to that mode. It is followed by the readjustment (coarsening), which ends up with the formation of 18R-type structure. Connection to the 14H-type part of the landscape is complicated and requires the driving force and time.

Our theoretical and numerical analyses of LPSO formation lead us to the following conclusions:

1. The LPSO is not a homogeneous phase but as a heterogeneous, two-phase structure of coexisting plates of hcp $\alpha-M g$ and fcc phase of the ternary $M g-X^{S}-X^{L}$ alloy.

2. Turing instability that is, existence of a finite wavelength linear-instability mode, is the origin of formation of a strictly periodic and highly synchronized LPSO structures.

3. Free energy difference between different types of LPSO structure-the most common of them are $18 \mathrm{R}$ and $14 \mathrm{H}-$ is the driving force for the transformation (e.g. $18 \mathrm{R} \rightarrow 14 \mathrm{H}$ ).

4. Coarsening of LPSO structure stops and does not lead to complete phase separation because of the interatomic barriers of the crystalline lattice.

5. The process of LPSO formation can be understood as a process of finding local minima and overcoming saddle points on the free energy landscape of the system.

\section{ACKNOWLEDGEMENT}

One of the authors (AU) acknowledges financial assistance provided by the awards HRD-1436120 from National Science Foundation and 70NANB14H012 from NIST U.S. Department of Commerce as part of the Center of Hierarchical Material Design at Northwestern University.

\section{LITERATURE}

1. E. Abe, Y. Kawamura, K. Hayashi, A. Inoue, Long-period ordered structure in a high-strength nanocrystalline Mg-1 at\% Zn-2 at\% Y alloy studied by atomic-resolution Z-contrast STEM, Acta Materialia 50 (2002) 3845-3857;

2. T. Itoi, T. Seimiya, Y. Kawamura,M. Hirohashi, Long period stacking structures observed in $\mathrm{Mg}_{97} \mathrm{Zn}_{1} \mathrm{Y}_{2}$ alloy, Scripta Materialia 51 (2004) 107-111. 
3. Y.M. Zhu, M. Weyland, A.J. Morton, K. Oh-ishi, K. Hono, and J.F. Nie, The building block of longperiod structures in Mg-RE-Zn alloys, Scripta Materialia 60 (2009) 980-983;

4. Y.M. Zhu, A.J. Morton, J.F. Nie, The $18 \mathrm{R}$ and $14 \mathrm{H}$ long-period stacking ordered structures in MgY-Zn alloys, Acta Materialia 58 (2010) 2936-2947;

5. Y.M. Zhu, A.J. Morton, J.F. Nie, Growth and transformation mechanisms of $18 \mathrm{R}$ and $14 \mathrm{H}$ in $\mathrm{Mg}-$ Y-Zn alloys, Acta Materialia 60 (2012) 6562-6572;

6. J.F. Nie, Y.M. Zhu, A.J. Morton, On the Structure, Transformation and Deformation of LongPeriod Stacking Ordered Phases in Mg-Y-Zn Alloys, Metal. Materials Trans. A 45A (2014) 33383348.

7. D. Egusa, E. Abe, The structure of long period stacking/order Mg-Zn-RE phases with extended non-stoichiometry ranges, Acta Materialia 60 (2012) 166-178.

8. J.-K. Kim, S. Sandlobes and D. Raabe, On the room temperature deformation mechanisms of a Mg-Y-Zn alloy with long-period-stacking-ordered structures, Acta Materialia 82 (2015) 414-423.

9. A.M. Turing, The chemical basis of morphogenesis, Phil. Transact. Royal Soc. B 237 (1952): 37

10. P. Grindrod, "Patterns and Waves" (Oxford, Clarendon Press, 1991).

11. A. Umantsev \& G. B. Olson, Modulation mechanism for first-order transformations with nonconserved order parameter, Phys. Rev. A 46 (10), Rapid Communications R 6132-R 6135, (1992).

12. A. Umantsev and G. B. Olson, Phase equilibria and transformations in adiabatic systems, Phys. Rev. E 48 (6), 4229-4249, (1993).

13. A. Umantsev, Field Theoretic Method in Phase Transformations, Springer, 'Lecture Notes in Physics', vol 840, 2012.

14. A. Umantsev, Continuum theory of interfacial segregation, Phys. Rev. B 64, 075419 (2001)

15. J.W. Cahn and J.E. Hilliard, J. Chem. Phys. 28, 258 (1958).

16. J.W. Cahn, Acta Metal. 9 (1961) 795-801.

17. A. Umantsev, Thermodynamic stability of phases and transition kinetics under adiabatic conditions, J. Chem. Phys. 96, 605 (1992)

18. J. Gröbner et al, Phase equilibria and transformations in ternary Mg-rich Mg-Y-Zn alloys, Acta Materialia 60 (2012) 5948-5962;

19. R. Masumoto, H. Ohtani, and M. Hasebe, J. Japan Inst. Metals, 73, (2009) 683-690

20. A. H. Cottrell, Dislocations and Plastic Flow in Crystals, Oxford, Clarendon Press, GB 1961. 
21. L.D. Landau and E.M. Lifshitz, Theory of Elasticity, Pergamon Press, Oxford, 1958.

22. J. Saal and C. Wolverton, Thermodynamic stability of Mg-based ternary long-period stacking ordered structures, Acta Materialia 68 (2014) 325-338.

23. http://en.wikipedia.org/wiki/IEEE floating point, June 2015.

24. J.D. Murray, Lectures on Nonlinear Differential Equations. Models in Biology, Oxford, Clarendon Press, 1977. 


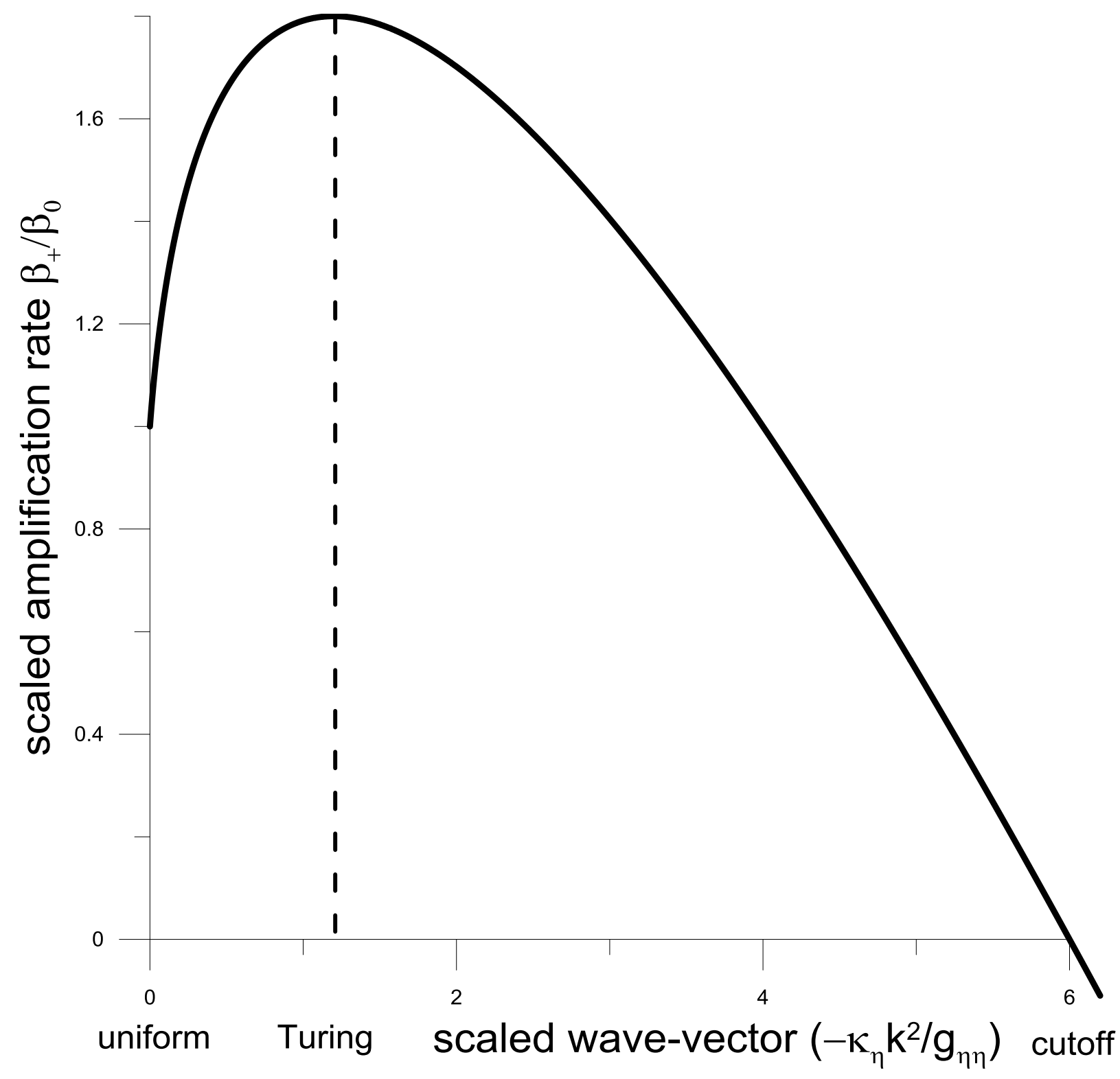

Figure 1. The positive branch of the amplification rate $\beta$ as a function of the square of the wave-vector for a system with $R=1$ and $A=5$. 


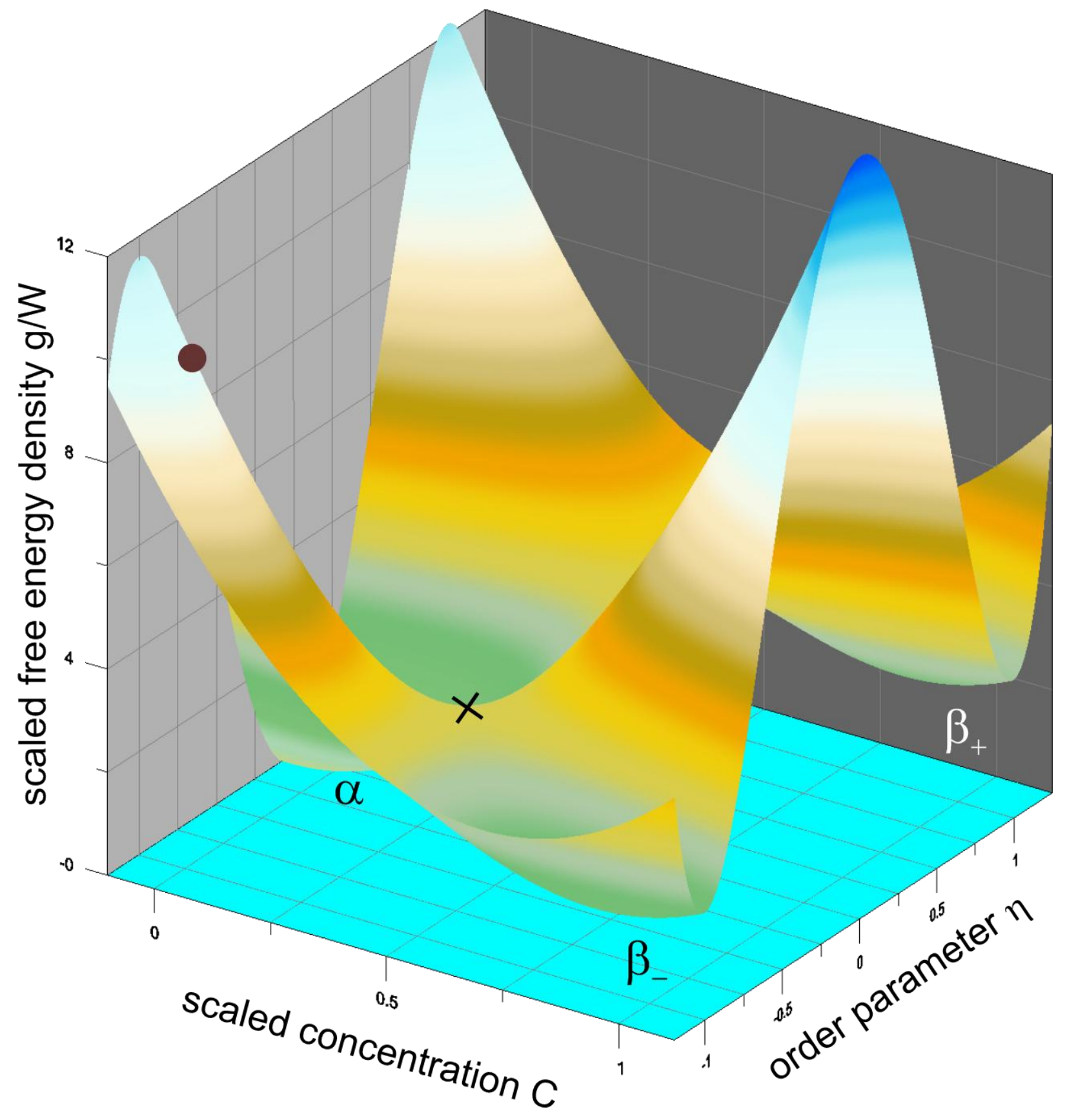

Figure 2. (Color online) The free energy density $g(\eta, X ; S)$ scaled with the barrier-height parameter $W$ as a function of the order parameter and concentration. Black cross-the saddle point; pink circle-energy density of the theoretical shear strength of the $\alpha$-phase. 


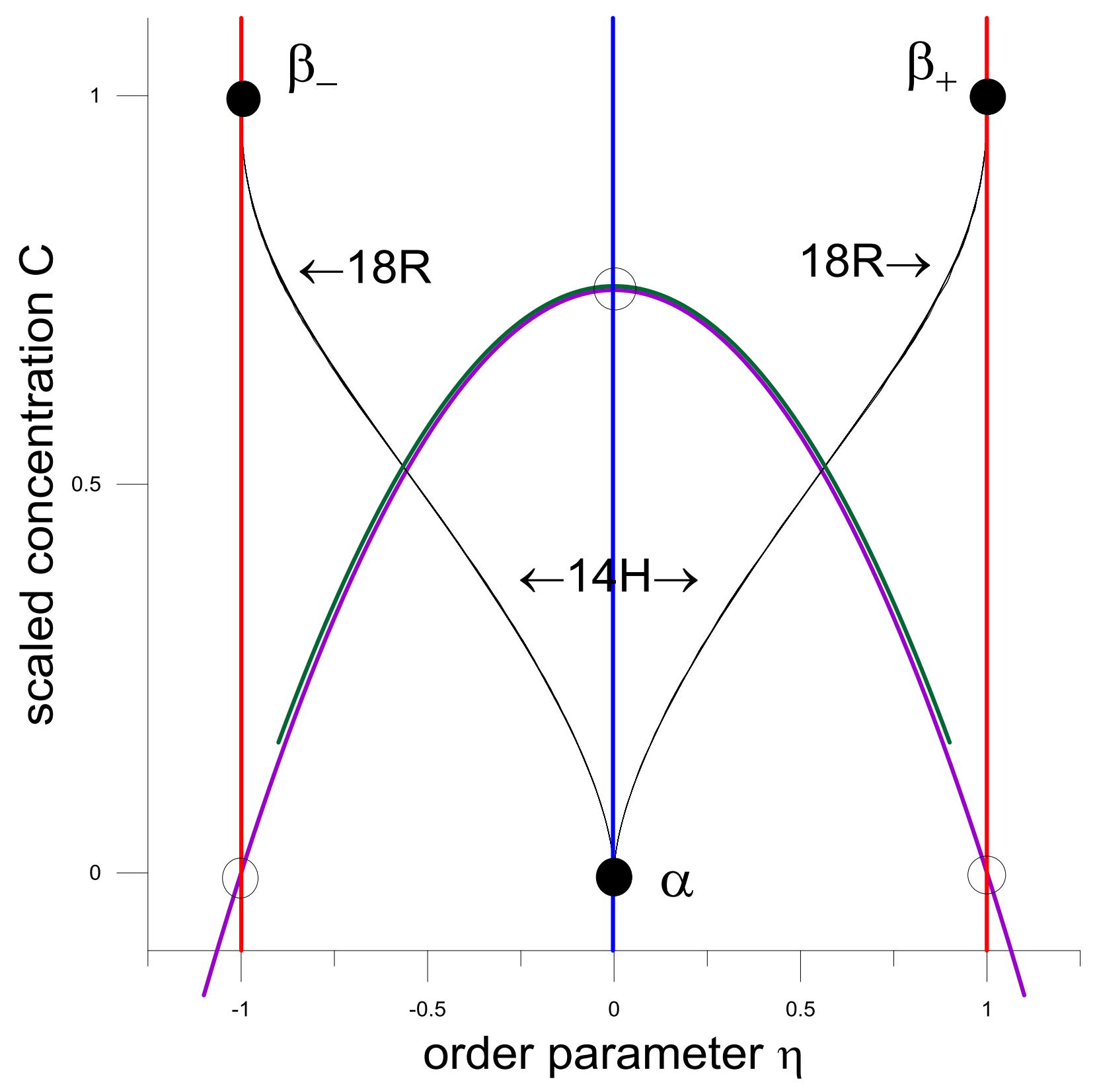

Figure 3. (Color online) Phase map of the system with $Q=1, K=1, R=1, \Sigma=0$ in the phase plane $(\eta, C)$. Red lines $-\beta_{+}$and $\beta_{-}$-phases; blue line $-\alpha$-phase; purple line - the barrier state; green highlight - the Turing mode; black lines-simulated 18R- and 14H-type LPSO structures. 


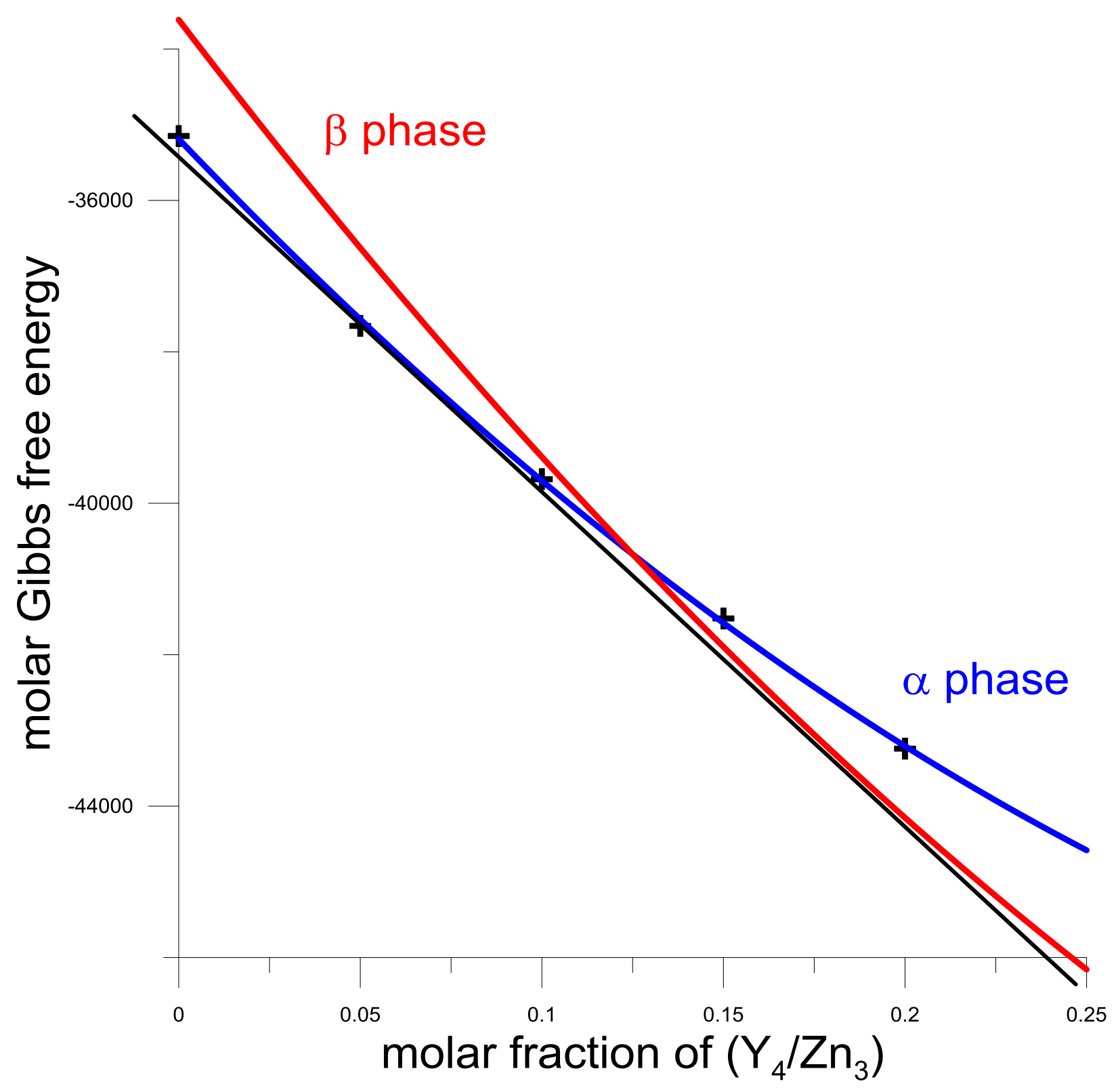

Figure 4. (Color online) The free energy of the hcp $\alpha-\mathrm{Mg}$ and fcc $\beta$-phase based on CALPHAD data [15, 16]. 


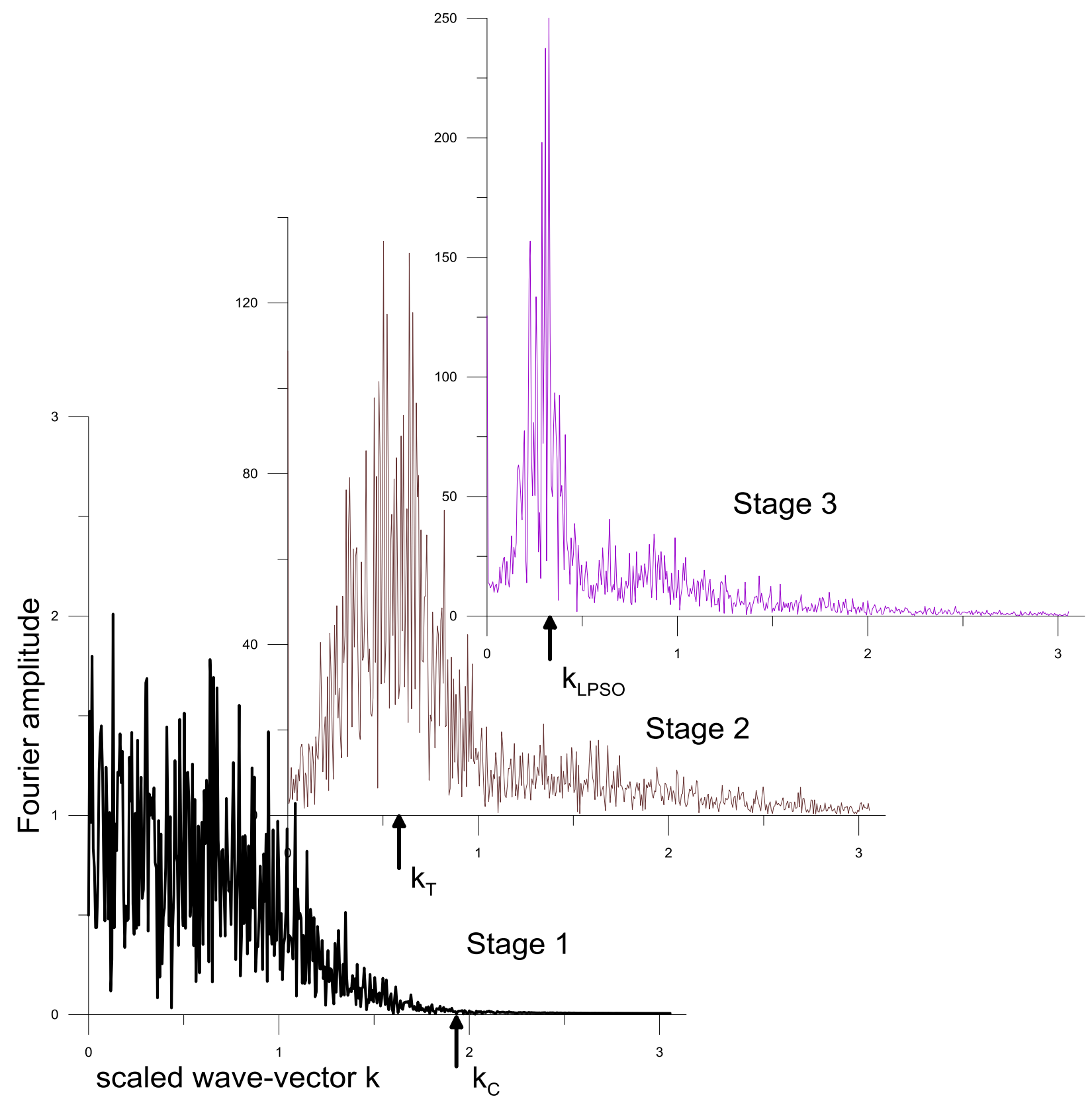

Figure 5. (Color online) Time sequence: (a)-time=1 (Stage 1), (b)-time=6 (Stage 2), (c) -time=5000 (Stage 3 ) of the Fourier transforms of the order parameter field of the system with $Q=1, K=0.125, R=1$, $\bar{C}=0.5$, and $\Sigma=0$. 

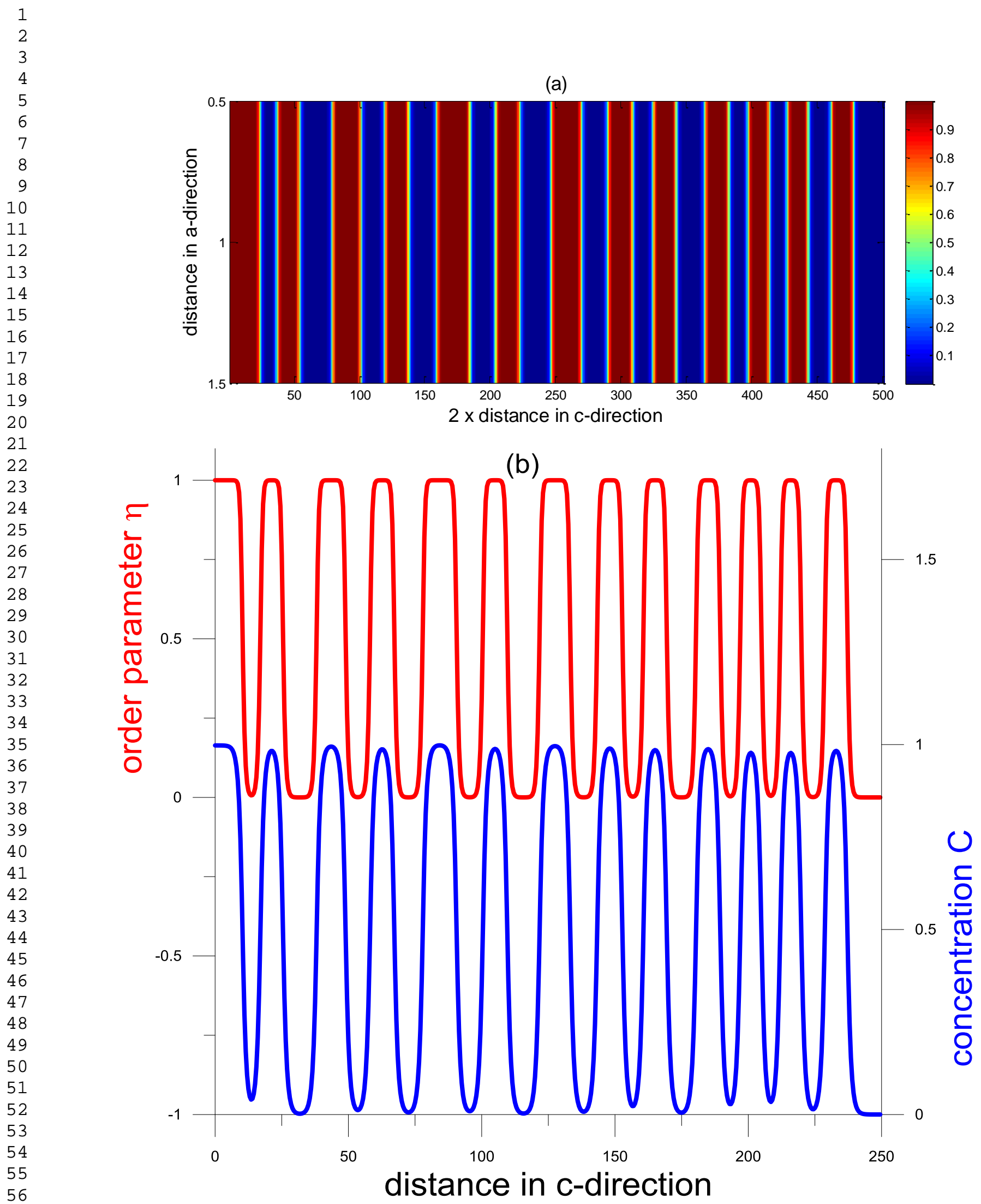

Figure 6. (Color online) 18R-type structure in the system with $\mathrm{Q}=1, \mathrm{~K}=1, \mathrm{R}=1, \bar{C}=0.5$, and $\Sigma=0$. (a)-Top view; (b)-structural and compositional profiles. Red-order parameter field; blue-concentration field. 
(a)
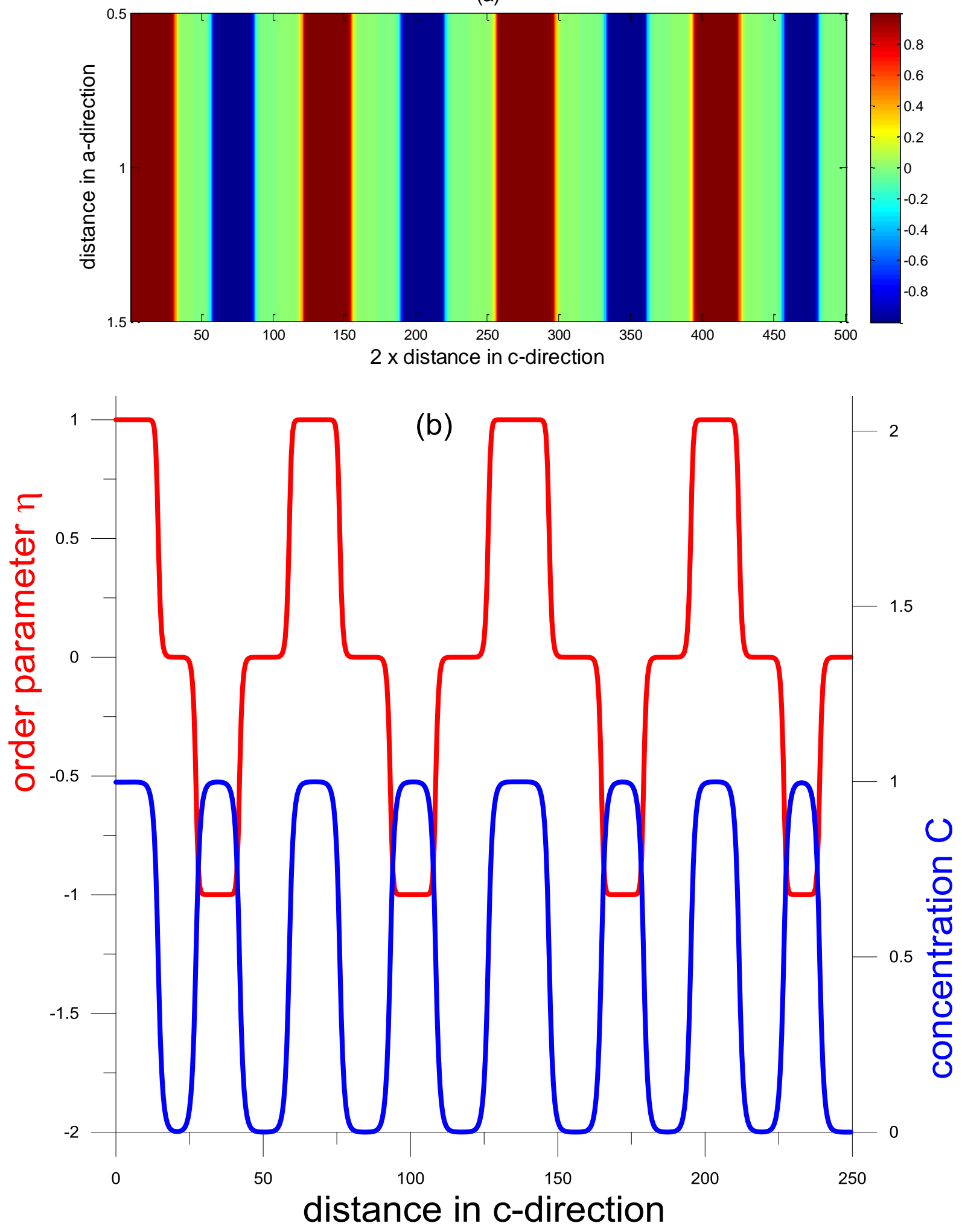

Figure 7. (Color online) $14 \mathrm{H}$-type structure in the system with $\mathrm{Q}=1, \mathrm{~K}=1, \mathrm{R}=1, \bar{C}=0.5$, and $\Sigma=0$. (a)-Top view; (b)-structural and compositional profiles. Red-order parameter field; blue-concentration field. 


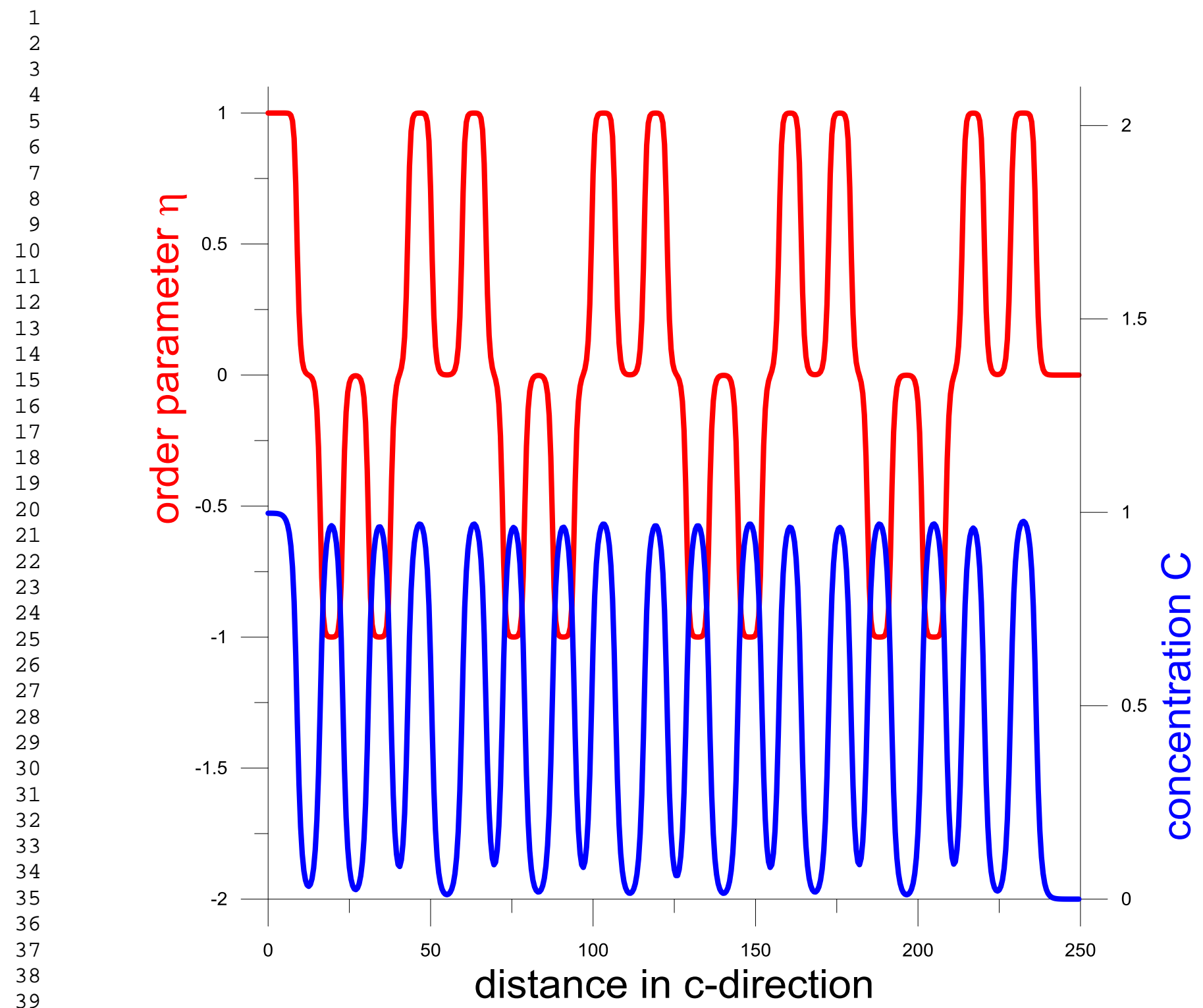

Figure 8. (Color online) Structural and compositional profiles of 18R-twin structure in the system with $\mathrm{Q}=1, \bar{C}=0.5, \mathrm{~K}=1, \mathrm{R}=1, \Sigma=0$. Red-order parameter field; blue-concentration field. 


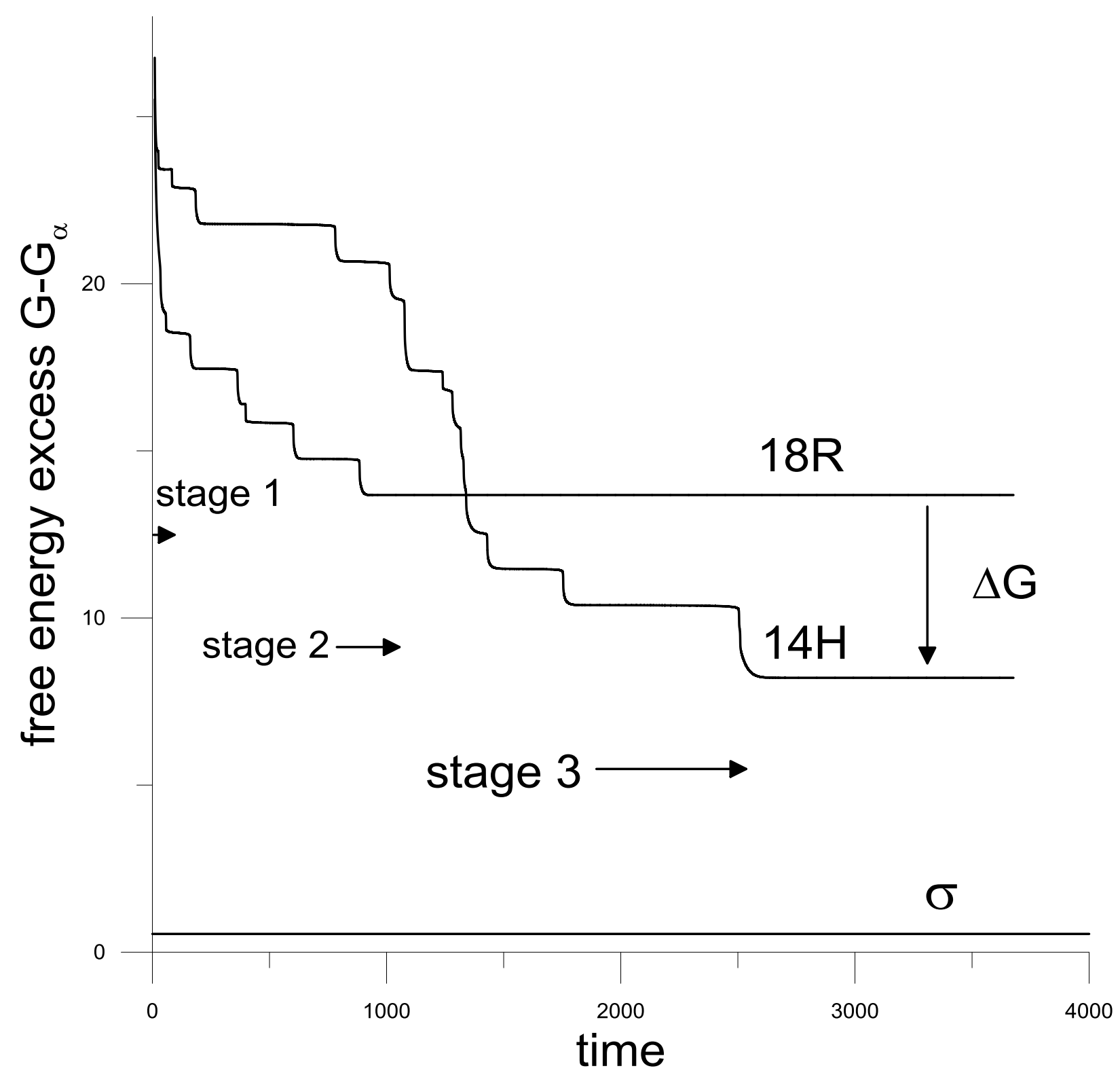

Figure 9. Evolution of the free energy excess of the $18 \mathrm{R}$ and $14 \mathrm{H}$ structures in the system with $\mathrm{Q}=1, \mathrm{~K}=1$, $\mathrm{R}=1, \bar{C}=0.5$, and $\Sigma=0 . \Delta \mathrm{G}$ is the driving force of the $18 \mathrm{R} \rightarrow 14 \mathrm{H}$ transformation. $\sigma$ is the interfacial energy of a completely phase-separated system. 


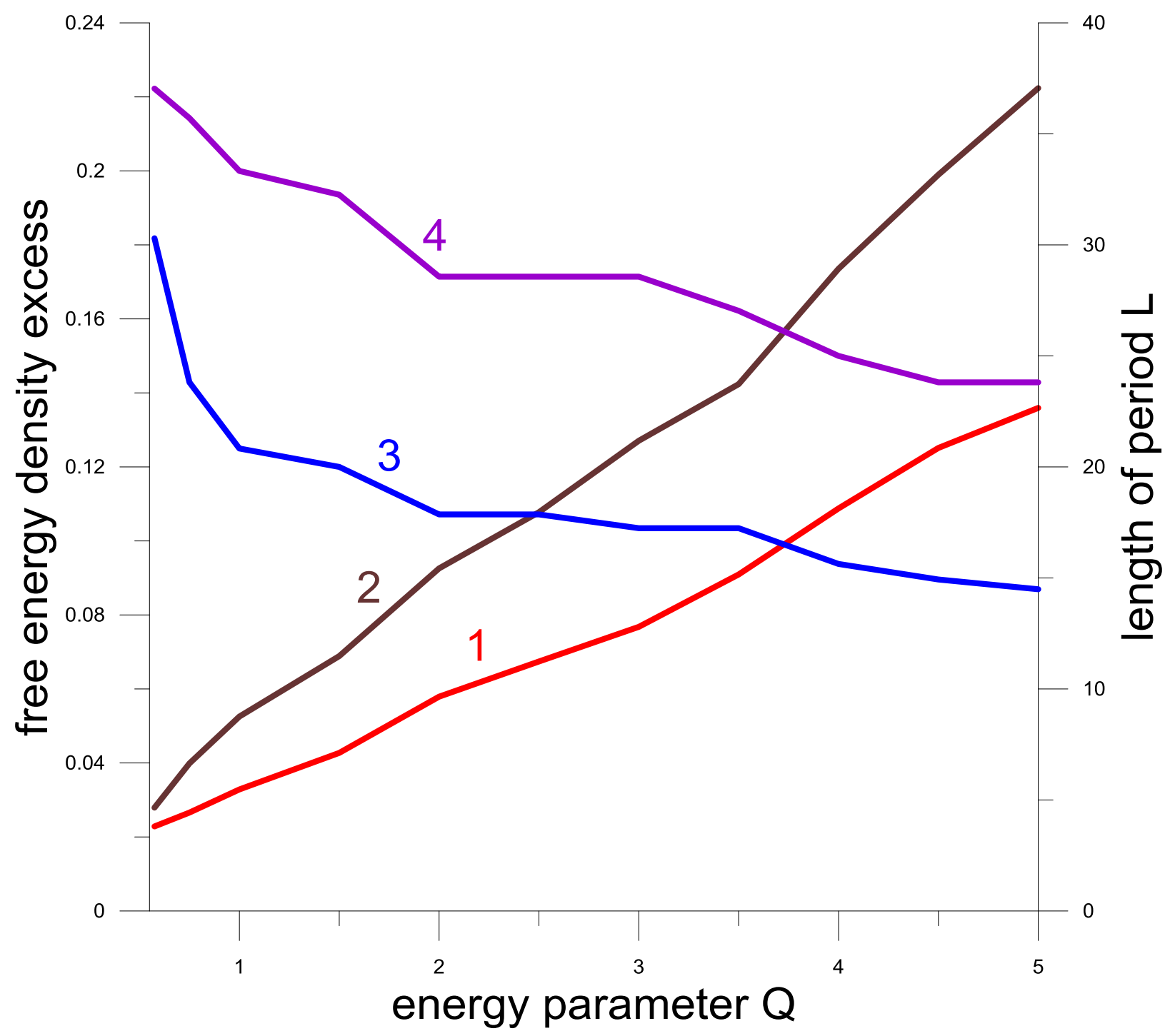

Figure 10. (Color online) Comparison of 18R-type and 14H-type structures in the systems with $\bar{C}=0.5$, $\mathrm{K}=1, \mathrm{R}=1, \Sigma=0$ and variable energy parameter $\mathrm{Q}$. 1 (red) -free energy density excess of $14 \mathrm{H}$-type; 2 (brown)-free energy density excess of 18R-type; 3 (blue)-length of period of 18R-type; 4 (purple)length of period of $14 \mathrm{H}$-type. 


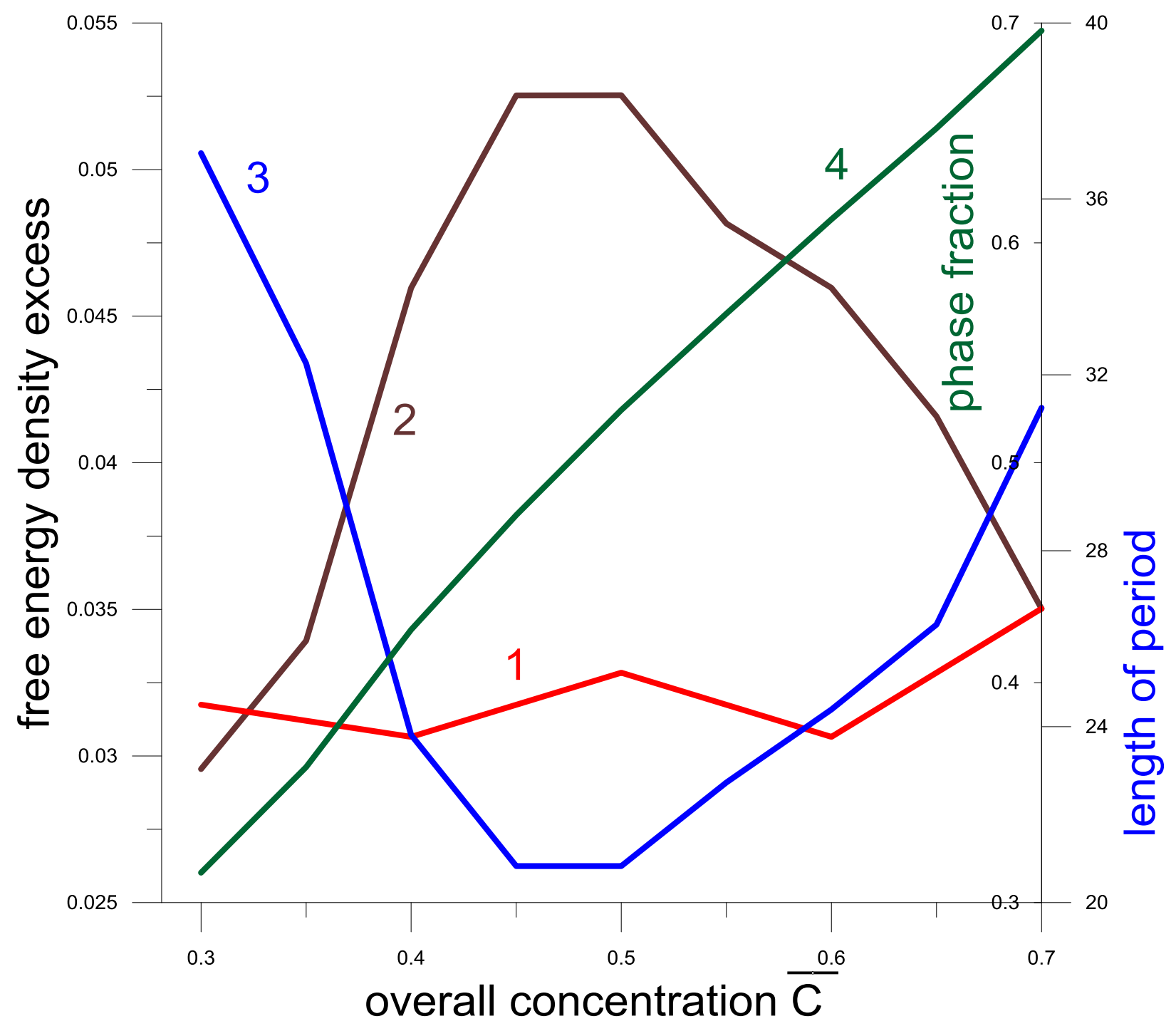

Figure 11. (Color online) Dependence on the overall concentration $\bar{C}$ of the LPSO structural characteristics in the systems with, $Q=1, K=1, R=1, \Sigma=0.1$ (red)-free energy density excess of $14 \mathrm{H}$-type; 2 (brown) -free energy density excess of 18R-type; 3 (blue)-length of period of 18R-type; 4 (green)- $\beta$ phase fraction of 18R-type. 


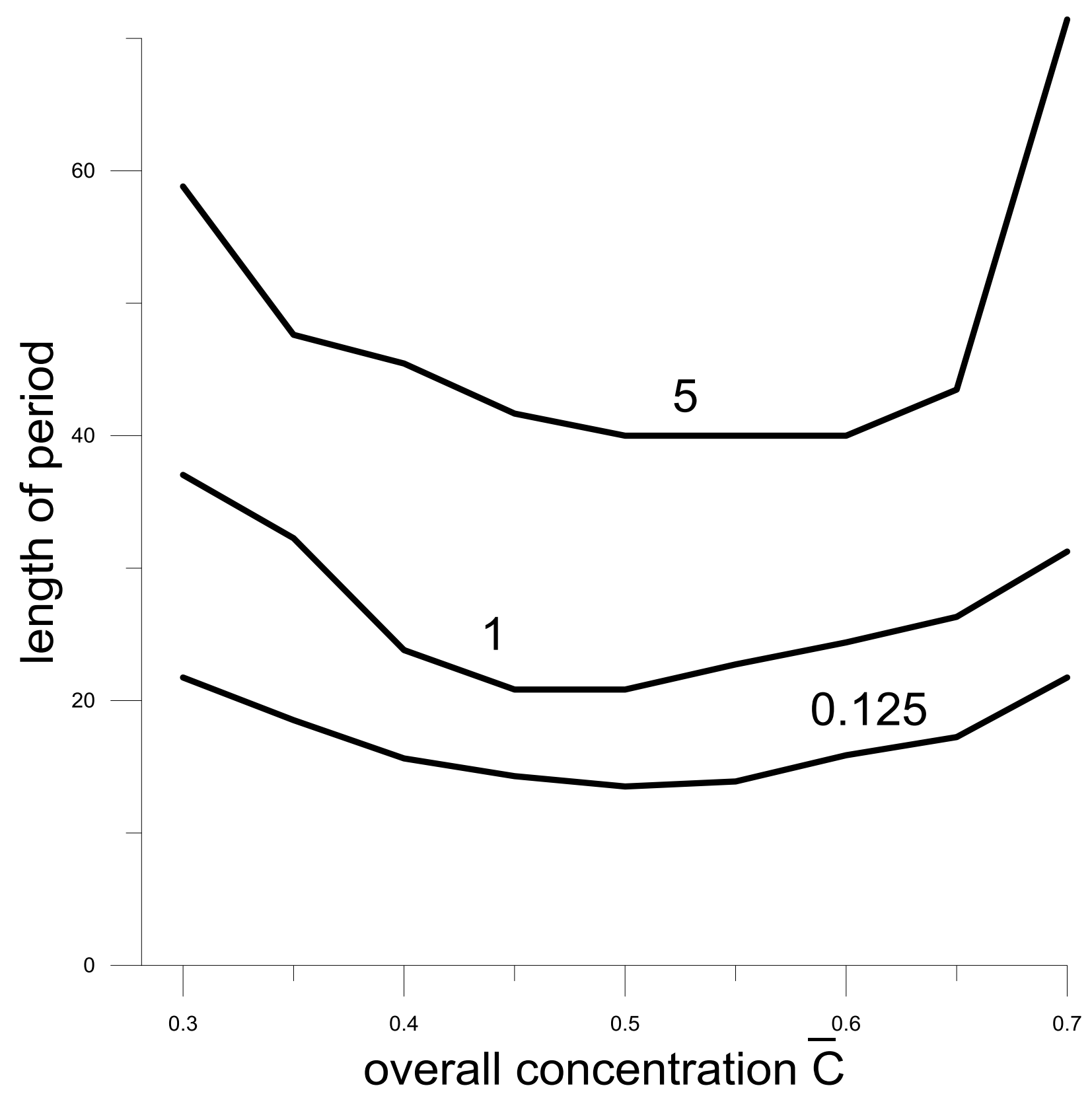

Figure 12. Dependence of the length of period $L$ on the overall composition $\bar{C}$ and gradient-energy ratio $\mathrm{K}$ in the systems with $\mathrm{Q}=1, \mathrm{R}=1$, and $\Sigma=0$. 


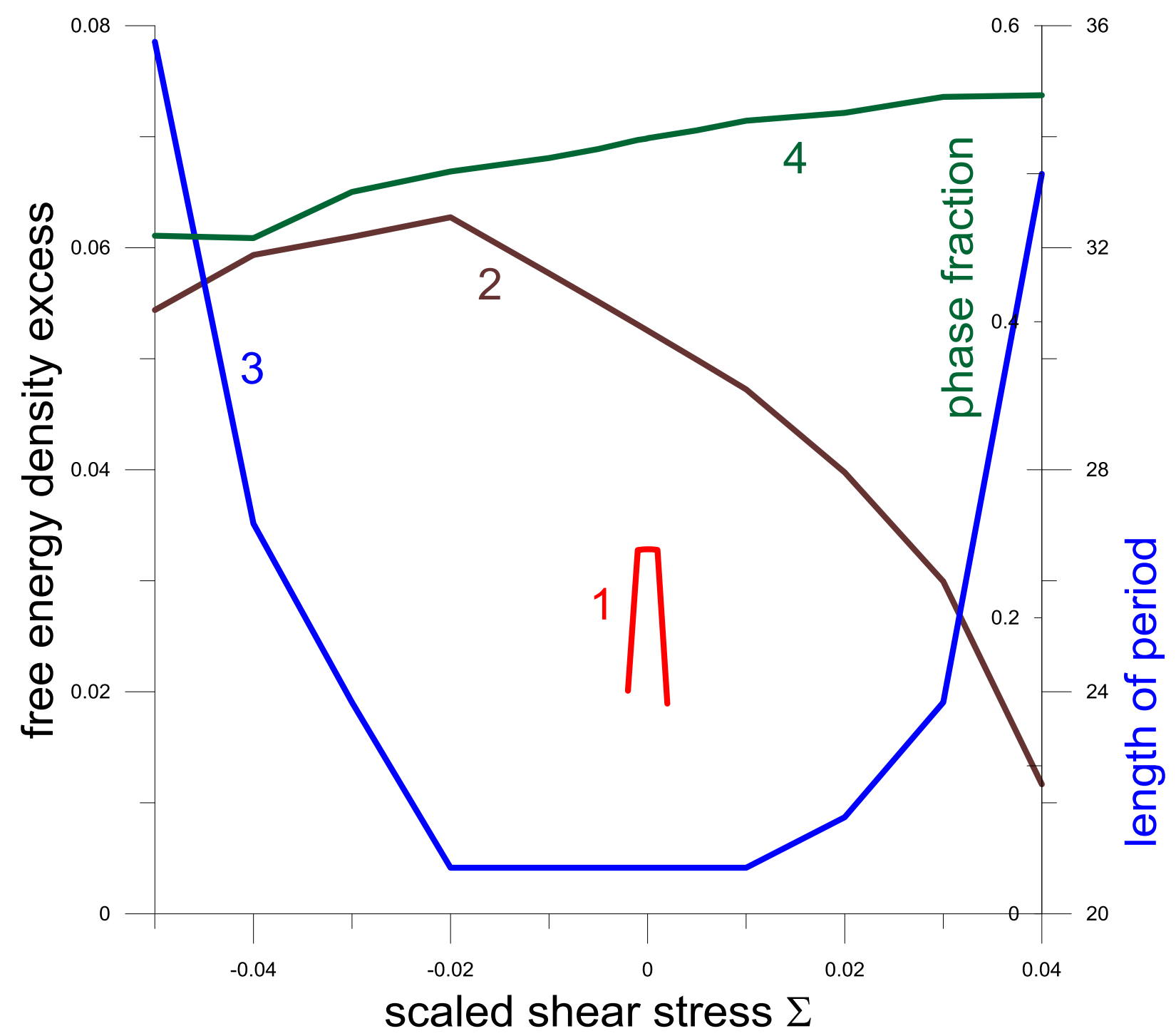

Figure 13. (Color online) Dependence on the applied shear stress $\Sigma$ of the LPSO structural characteristics in the system with $Q=1, K=1, R=1, \bar{C}=0.5$. 1 (red)-free energy density excess of 14 H-type; 2 (brown)-free energy density excess of 18R-type; 3 (blue)-length of period of 18R-type; 4 (green)- $\beta$ phase fraction of 18R-type. 


\title{
Formation of Long-Period Stacking Fault Structures in Magnesium Alloys
}

Alexander Umantsev

Department of Physical Chemistry, National University of Science and Technology MISiS, 4 Leninsky Ave, 119049 Moscow, Russian Federation and Department of Chemistry and Physics, Fayetteville State University, 1200 Murchison Rd., Fayetteville, NC 28301

\section{aumantsev@uncfsu.edu}

Machiko Ode

Computational Materials Science Unit

National Institute for Materials Science

Tsukuba, Ibaraki, 305-0044 JAPAN

ODE.Machiko@nims.go.jp

\begin{abstract}
We developed a Ginzburg-Landau model of long-period stacking order (LPSO) lamellar structure observed in magnesium alloys. Contrary to other models, we do not treat LPSO as a homogeneous stoichiometric phase but as a chemically modulated heterogeneous structure of parallel plates of two phases with different compositions and degrees of ordering. The Turing instability that is, existence of a finite-wavelength linear-instability mode was identified as the origin of formation of LPSO structure. The process of LPSO formation can be understood as a process of finding local minima and saddle points on the free energy landscape of the system. The model explained transformation between the two most common LPSO structures, $18 \mathrm{R}$ and $14 \mathrm{H}$, and their general resistance to coarsening. The model can be used for the design of new LPSO materials.
\end{abstract}

(a)

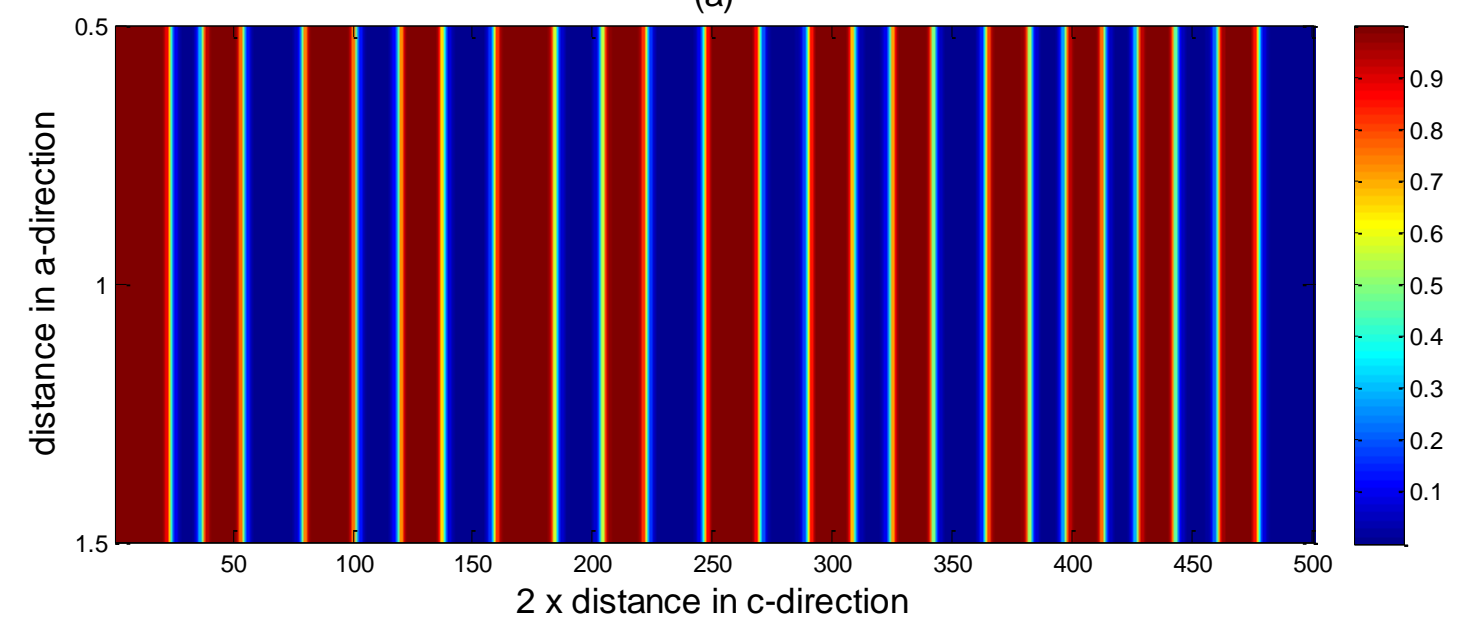




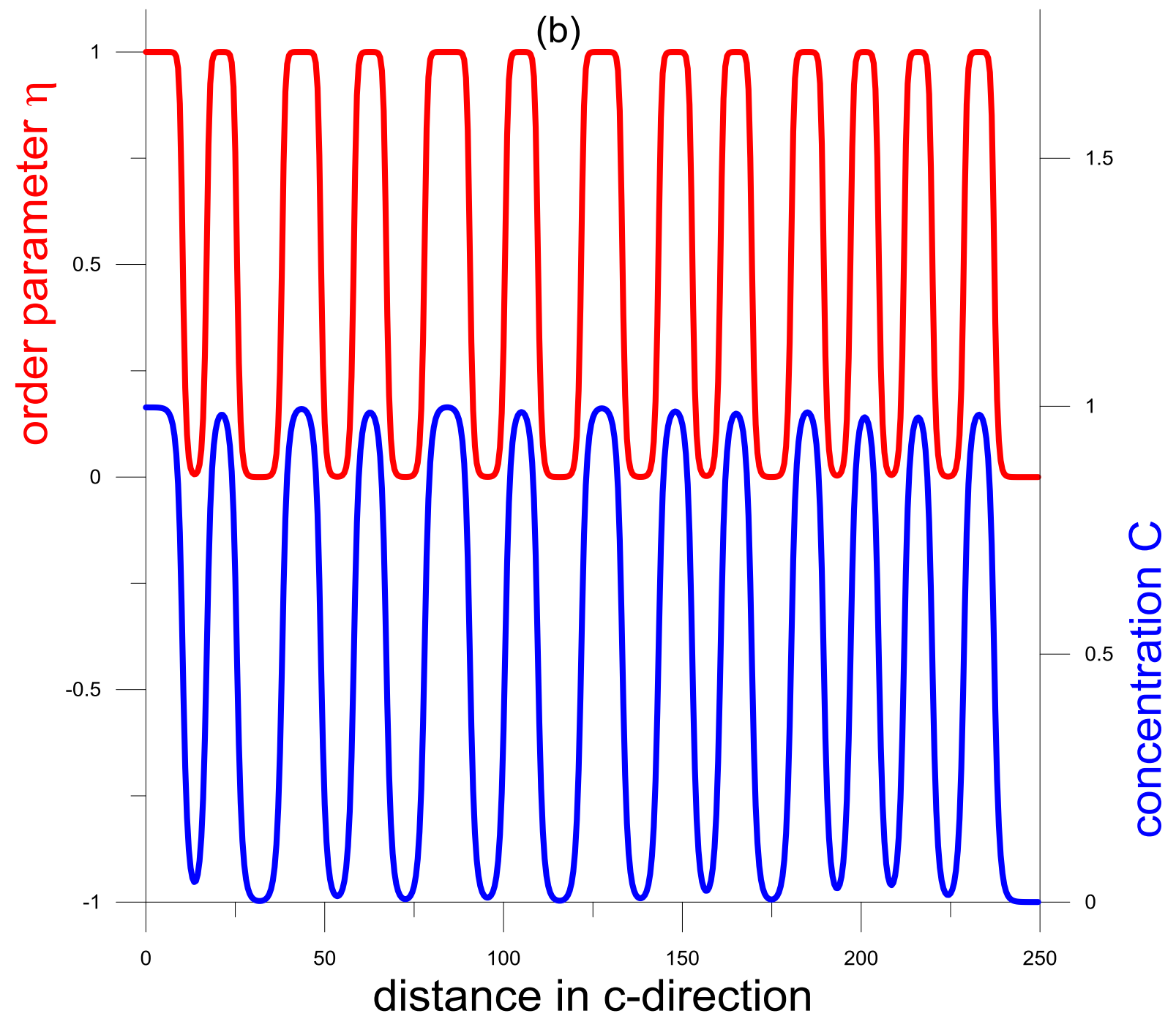

